\title{
Insight into the Role of Photoreception and Light Intervention for Sleep and Neuropsychiatric Behaviour in the Elderly
}

\author{
Katharina Wulff ${ }^{1 * *}$ and Russell G. Foster ${ }^{1}$ \\ ${ }^{1}$ Sleep and Circadian Neuroscience Institute (SCNi), Nuffield Laboratory of Ophthalmology, University of Oxford, UK
}

\begin{abstract}
Light exerts influences on many physiological and behavioural functions in humans. These functions can be described as image-forming (IF) and non-image forming (NIF) visual processes, both originating in the retina of the eye. Image-forming refers to vision; the process of detecting and distinguishing shapes and colour of objects. Non-image forming refers to detecting level of light intensity or brightness of ambient space, which affects basal physiology such as cycles of rest and activity or the endocrine system. Rod and cone photoreceptors in the outer retinal layer are most important for image-forming vision, while non-image forming functions depend upon additional input from the photopigment melanopsin, which is expressed in retinal ganglion cells (RGC) that makes these cells photosensitive (pRGC). Projections of these pRGCs convey light-induced electrical impulses to a number of brain regions. Visual acuity and colour contrast naturally diminishes with age but dementia often has major effects on the visual processing systems, which impact on the quality of life. The ability of humans to manipulate their light exposure has the immediate potential to either create problems with human physiology (as in shift workers) or to compensate physiological disadvantages (of IF and NIF visual impairment). This mini-review describes the impact of aging on the function of the eye with respect to non-image forming effects of light, summarises light intervention studies for sleep and neuropsychiatric symptoms and considers implications from photoreceptor-weighted light intensities for biologically effective light intervention and lighting solutions for patients with dementia.
\end{abstract}

Keywords: sleep, circadian, melanopsin, photoreception, retina, visual impairment, Alzheimer, cognitive impairment.

\section{Non-image forming vision and sleep timing}

Biological timekeeping of the sleep-wake cycle is needed for people's well-being. Sleep timing is regulated by complex, dynamically changing circadian and homeostatic processes. In humans, the 'master body clock' consists of two clusters of pacemaker neurons located within a small anterior hypothalamic structure that sits on top of the optic chiasma - the suprachiasmatic nuclei ( $\mathrm{SCN})$. Although they constitute the main body clock, the $\mathrm{SCN}$ is not the only one. Most, if not all, cells of the body have the capability to generate autonomous $24 \mathrm{~h}$ rhythms of activity [1]. The mammalian retina has at least one, but probably a network of circadian clocks, controlling ocular function [2]. The daily rhythms of such 'peripheral clocks' interact with the SCN and as a result, the 'day within' arises from a complex network of coupled circadian oscillators. However, if this timing system is to have any adaptive value, then it needs to be locked-on (entrained) to the environmental light/dark cycle to ensure that internal and external time coincide. The retina detects the changes in light exposure from waking to bed time and sends these signals along the retinohypothalamic tract to the SCN allowing entrainment.

*Address correspondence to this author at the University of Oxford, Sleep and Circadian Neuroscience Institute, Sir William Dunn School of Pathology (OMPI), South Parks Road, Oxford, OX1 3RE; Emails: katharina.wulff@eye.ox.ac.uk
It is therefore clear that the eye is not only important for what we normally think of as vision, which involves forming a detailed image of the world around us. It is also involved in other, non-image forming processes such as the synchronising of the body's rhythms, for which detailed information about the image is not required. What is important is a measurement of the absolute light level.

It has long been known that rods and cones underlie the image-forming (IF) functions of the eye. However, it was unclear which photoreceptor(s) was responsible for the eye's non-image forming (NIF) responses and the input to the $\mathrm{SCN}$. Was it rods or cones or perhaps both? The answer surprised everyone as it was, 'neither'. Circadian rhythms in mice that had been genetically altered to have no functional rods and cones continued to oscillate, with activity and hormone cycles perfectly aligned to the periods of light and darkness [3]. The only possible conclusion was that there was another non- rod, non-cone photoreceptor that was supplying information about ambient light levels to the SCN. This novel photoreceptor in mammals is a subset of directly photosensitive retinal ganglion cells (pRCGs) containing the photopigment melanopsin [4-11]. These cells, which make up about $1 \%$ of all ganglion cells in the human retina, can respond to light without any inputs from the rods or cones, but also receive contributions from rods and cones (Figure 1, insertion). Human rods contain a visual pigment absorbing maximally at $498 \mathrm{~nm}$ and we have 3 cone types maximally 
sensitive near 420, 530 and 560nm [12], with overall photopic sensitive at around $555 \mathrm{~nm}$. The sensitivity of melanopsin is in the "blue" part of the spectrum around $480 \mathrm{~nm}$, perhaps tuned to the blue-enriched wavelengths of light across the dome of the sky at dawn and dusk [13]. At twilight, the immediate horizon is red/orange, but the sky is blue - peaking around 460-480nm [14].

The announcement in 1999 of the existence of a third photoreceptor type in the mammalian retina [3] was met with widespread scepticism. People could not imagine why they were needed; why not use the traditional photoreceptors to inform the SCN about light levels? The answer is that the properties of the photoreceptors required for the two functions of the eye, IF and NIF vision, are largely incompatible. To detect detailed information for the highresolution IF vision, a photoreceptor needs to adapt rapidly, while one that measures the amount of light in the environment for NIF vision needs to keep responding for much longer. Thus, while the cones and rods adapt very quickly to changes in light stimuli, which is necessary for detecting images, pRGCs cells show sustained responses to light and in so doing pRGCs can continue firing for several minutes after a light stimulus [15]. Thus, the existence of separate photoreceptor classes for IF and NIF vision is of advantageous adaptive value and not surprising (Figure 1).

There are at least five different morphological types of pRCGs that project to different parts of the brain [16-18]. The precise details of where they project, how these projections overlap, and how their responses to light differ is still being unraveled [19]. However, those pRGCs that project to the SCN, will encode NIF information in a slightly different manner compared to those pRGCs that project to the olivary pretectal nuclei and control the pupil responses, or those that project to the ventro-lateral preoptic nuclei and regulate sleep and arousal [20,21].Also, pRGCs have a role to play in conscious brightness perception through projections to the dorsal lateral geniculate nuclei $[22,23]$

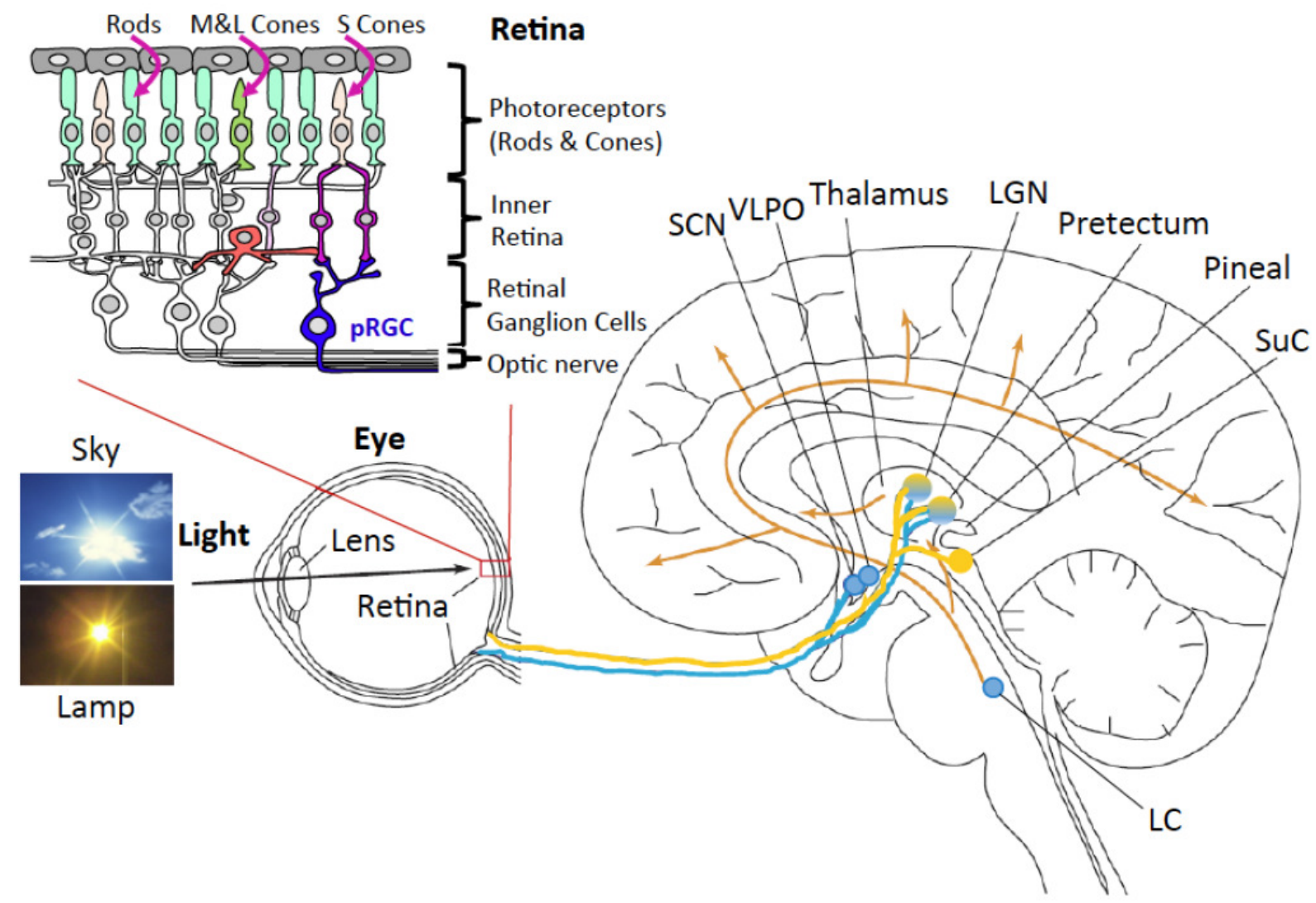

Figure 1. Schematic of the pathways for light-induced activation of brain areas involved in image-forming (IF) and non-image forming (NIF) functions. Insertion: Light exposure at the level of the retina activates rod and cone photoreceptors as well as melanopsin-containing photoreceptive ganglion cells (pRGCs) at their respective range of wavelengths and in proportion to the intensity of illumination (see text). The subset of pRGCs also receives indirect input from rod and cones via bipolar (light \& dark purple) and amacrine (red) cell networks. pRGCs project from the eye to various brain regions, involved in NIF functions (blue projection) such as suprachiasmatic nuclei (SCN - the master circadian clock); the ventrolateral preoptic nucleus (VLPO), important for switching between sleep and wake, the pineal gland (place of light-dependent melatonin production), and the locus coeruleus (LC), implicated in the regulation of arousal. pRGCs also share input with RGC projections (yellow) to brain regions involved in IF visual functions, such as the lateral geniculate nucleus (LGN), pretectum and superior colliculus $(\mathrm{SuC})$. Light also stimulates the ascending arousal system (orange) and enhances alertness through as yet unidentified pathways. Adapted from Lockley and Gooley (2006). Curr Biol;16:R795-7. 


\section{Age-related ocular changes affecting the light sensitivity and visual pathways}

In order to obtain proper visual sharpness, the average 60-year old person needs two to three times the light of a 20-year old, and an 86-year old person may require five times the lighting levels. These differences in light intensities are due to age-related lens yellowing, opaque cataract or pupil narrowing [24-26], creating a decline in retinal illumination, which makes the effective adaptation luminance intensity lower for older adults [27]. Therefore, older adults generally require better contrast and higher task luminance to obtain the same visibility level as a younger person.

Through reduction in light transmission to the retina by the lens, increased spectral absorption, particularly at the blue end of the spectrum, and increased lens fluorescence [28], NIF light sensitivity may be impaired, which may manifest as a change in sleep-wake patterns or sleep disruption, but equally, compensatory mechanisms may maintain sensitivity to light [29]. This reduction in photic input increases further when opaque cataracts, the clouding of the lens, become more mature. Replacing a cataractous lens with an artificial, intra-ocular lens allows much more light to enter the eye and results not only in subsequent improvement in visual acuity but also in sleep [24, 30]. Preliminary studies report that cataract surgery in Alzheimer's patients also reduces decline in memory and executive function in addition to improved visual acuity and sleep, but the light-transducing pathways involved have yet to be linked [31].

In patients with Alzheimer's Disease (AD), a number of visual changes have been reported, including loss of visual acuity, diminished contrast sensitivity and disturbances in smooth and saccadic eye movements [32]. Visual sensory impairments in Alzheimer's patients were believed to reflect cortical disturbances rather than $\mathrm{AD}$ associated optic neuropathy. However, pathological changes have been observed in the visual system from the eye to the brain and are likely to involve a combination of both ocular and neural pathways. Using retinal photography and optical coherence tomography, patients with AD showed reductions in the number of ganglion cells and thickness of the retinal nerve fibre layer [33]. This has also been assumed from postmortem retinae of patients with $\mathrm{AD}$ [34]. A consistent change affecting small and large axons is the decline in the density of optic nerve fibres $[35,36]$. Whether this reflects a reduction in retinal ganglion cells or originates from retrograde degeneration of the visual cortex, or both, is currently unclear. Furthermore, supranuclear cataracts were found in lenses of patients with $\mathrm{AD}$, with amyloid- $\beta(\mathrm{A} \beta)$ specifically accumulated in the cytosol of lens fibre cells that could promote region-specific lens protein aggregation [37]. Therefore, it is particularly important to carry out a full ocular examination and a motility test of eye movements in patients with dementia because these visual impairments may contribute or exacerbate to sleep-wake disturbances.

\section{Daylight and its effects on human circadian physiology}

Before the invention of electrical light, humans lived their lives for thousands of years exposed to changing spectra of light in the morning, the late afternoon and evening. Nowadays humans spend, however, little of their lives exposed to natural light, instead electrical light extends the period of wakefulness into the late evening, thereby condensing sleep. A study in New England found that older people (mean age 66 years) spent on average $38 \%$ of their waking day in light levels above 100 lux and $15 \%$ in light levels above 1000 lux (likely to be outdoor levels of light), whereas young subjects (mean age 24 years) spent only $27 \%$ of their waking day in light exceeding 100 lux and only $9 \%$ in light levels above 1000 lux [38]. This observation raises the question, whether differences in the duration and level of light exposure between young and old subjects may contribute to age-related sleep-wake timing differences.

A recent cross-over study compared physiological responses to light in young adults when living in constructed buildings with access to electrical light and then again living exclusively outdoors exposed to daylight only [39]. Living in constructed buildings resulted in less sunlight to people and prolonged electrical light after sunset, which correlated with later waking activities and a later melatonin rhythm. This implies that the circadian clock responds to the electrical light of high colour temperature in the evening by shifting its activity to this artificial light-dark cycle [40]. Under outdoor conditions, people with originally late waking activities advanced their phase in response to the natural light-dark cycle. This daylight-associated phase advance was also found in old people in the study by Scheuermaier et al. (2010), where the older people with more daylight exposure showed an one-hour advance in bedtime and get-up time compared to the younger people. Evidence that this phase difference reflects photoentrainment of the SCN and not masking (direct enhancement of activity by light, not mediated via the SCN) comes from another study in elderly people. Increased exposure to natural daylight in these elderly correlated with an increase in nocturnal melatonin secretion [41]. Increasing the time from 37 to $124 \mathrm{~min}$ in natural daylight $(\mathrm{N}=192$, mean age $=69.9 \mathrm{yrs})$ increased night-time urinary 6-sulfatoxymelatonin excretion by $13.0 \%$ (6.8 to $7.7 \mu \mathrm{g})$, suggesting a strengthened biological timing signal.

While these studies showed that NIF effects of light depend on the duration and time-of-day of light exposure, experimental studies have also shown that the responses vary with the quality and intensity of light. The greatest sensitivity to light occurs during the habitual night time, when there is typically little light exposure [42, 43]. The most useful tools to predict optimal light intervention for restoring appropriate timing of the biological clock are doseand phase-response curves derived from circadian marker rhythms (advance/delay) in response to light. 


\section{Aging effects on dose- and phase-response relationships to light}

Requirements for light therapy are likely to differ for old individuals due to reduction in light transmission of the aged lens, changes in pupil adaptation and a general agerelated earlier phase and lower amplitude in their melatonin rhythm [44]. The relationship between white light intensities (dose) and circadian responses, such as phase shifts in melatonin mid-point and suppression of melatonin amplitude, in single $6.5 \mathrm{~h}$ light stimuli in the late evening was tested in extremely healthy predominantly older men under laboratory constant routine conditions [45]. Ageing was associated with a reduced capacity to suppress melatonin at any light intensity. Furthermore, the progressive phase-delay in the melatonin midpoint in response to low-to-moderate levels of light (approximately 50 to 1,000 lux) was slower in older people. The phase response to maximum levels of light (9,500 lux) did not differ between old and young people. That phase-shifting responses to light at higher intensities is not affected by age was also found in another study comparing 2 hours of light exposure at either 2000 lux or 8000 lux broad spectrum light administered at three different time-points in young versus older individuals [44]. These results indicate age-related differences in the magnitude of melatonin suppression by light and in the phase-shift response to low-intensity light exposure. Other studies found wavelength-dependent changes in melatonin suppression in older people, with a reduced response to shorter wavelength $(\sim 460 \mathrm{~nm})$ but not to longer wavelength $(550 \mathrm{~nm})$ light[46], which might be explained by greater blue light absorption by the aged crystalline lens.

\section{Dose- and phase response relationships to light for sleep and psychiatric symptoms}

Investigations into changes in sleep quality and mood in responses to light interventions can complement understanding of the dose- and phase effects of light on wellbeing. Most robust evidence of an effect of the level and timing of light on mood comes from randomised trials under controlled conditions in people with Seasonal Affective Disorder (SAD) and unipolar depression. The diagnosis of SAD is based on episodes of depression in at least two subsequent years during months of short photoperiod (winter) and remission of symptoms during long photoperiod (summer). While depressive symptoms are similar to those of unipolar depression, SAD is atypical in that symptoms include craving sweet things, increased appetite, weight gain, and increased sleepiness [47]. Reports show a dose-response relationship for morning light in SAD patients for typical but not atypical symptoms, with strong light $(6,000$ lux and more) being more effective than medium light (1,700-3,500 lux) or dim light (600 lux and less) [48]. Correspondingly, time of day evaluation of bright light therapy in unipolar depression showed, that morning exposure was more effective than at any other time of day, and this was equal between groups with and without concomitant drug therapy [49]. These studies indicate that light of high intensity applied in the morning has therapeutic effects specifically on typical mood symptoms. This was corroborated by studies, showing that bright light therapy (10,000 lux) in comparison to medium (4,000 lux) or dim light (50 lux), applied in the morning as adjunct treatment, increased the antidepressant effects of SSRIs such as sertraline and citalopram in patients treated for depression [50, 51]. However, the efficacy of light therapy as an adjunct was not confirmed in a metaanalysis on bright light therapy for mood disorders [52]. Light therapy in form of a dawn simulation in non-seasonal depression showed similar effect sizes to bright light but here no dose-response relationship was carried out [52]. The effect sizes for light therapy are equivalent to those in antidepressant pharmacotherapy trials[52].

\section{Light intervention in healthy elderly and people with dementia}

When studying a group of senior individuals with good physical and mental health, there was negligible seasonal variation in mood and behaviour and very little depressed feeling [53]. Moreover, when exposed to additional bright light, the intervention tended to make these healthy elderly individuals more irritable, anxious, and agitated whilst sleep was not affected, suggesting that bright light is not beneficial for healthy elderly individuals unaffected by mood. Good quality trial evidence for light therapy in treating sleep disturbances (no comorbidities) in the healthy population over 60 years are currently lacking and as a result, no conclusions for the effectiveness of light treatment could be determined [54].

Based on pooled studies, the effectiveness of increased indoor light intensity in residential care homes as a treatment for sleep problems, such as sleep onset latency, reduced sleep efficiency and night-time awakenings, is currently ambiguous with the magnitude of change varying considerably between studies [55]. Forbes et al. (2014) evaluated bright light interventional studies in a metaanalysis and reported no evidence that bright light reduces sleep onset latency, increases total night-sleep duration (neither after six weeks nor after two years) or improves sleep efficiency and decreases night-time activity. However, single studies in visually intact patients diagnosed with dementia report improvements in day-to-day rest-activity patterns as a result of increased indoor illumination [56, 57]. A double-blind, long-term clinical trial[58], also included in Forbes' meta-analysis, has shown a modest benefit of bright light therapy in improving day-time activity and some cognitive and non-cognitive symptoms of dementia. A systematic review of 10 comparative studies and clinical trials concluded that increased intensity of light (above 2,500 lux) in the morning or all day showed a trend in improving night-time sleep and in reducing day-time sleepiness [59].Similar results were published recently in a metaanalysis comprising 53 studies ( $\mathrm{N}=1154$ subjects) examining effects of light therapy on sleep in general and on specific types of sleep problems related to (i) circadian rhythm sleep disorders [CRSD], (ii) insomnia, and (iii) those related to dementia[60]. Detailed analyses uncovered Hedges' g effect sizes of small to moderate magnitude in treating sleep 
problems with light therapy. A dose-response effect was noticed for insomnia groups with CRSD, in which stronger light intensities showed larger effect sizes. Study design had moderating effects, such that smaller effects were generally reported from randomised clinical trials (RCT), (e.g. CRSD overall: $\mathrm{g}=0.41, \mathrm{p}<0.01$ versus CRSD RCT: $\mathrm{g}=0.27, \mathrm{p}=0.02$ ) and from studies with more men, in particular for dementiarelated sleep problems. Effect sizes were generally smaller for sleep timing variables than for subjective sleep complaints, such as insomnia symptoms. Effect sizes varied substantially between studies and between outcome variables. Negative results or opposing effects for at least one outcome were found in up to $53 \%$ of the studies. Overall, light therapy proves to be effective for different sleep problems but the technical aspects and optimal use still needs to be investigated.

In two recent light exposure studies in care homes (published in this issue), an interesting discrepancy has arisen between findings suggesting that imposed blueenriched light could detrimentally increase night-time activity while beneficially lower day-time anxiety [61], and those that suggest that self-inflicted greater power of light during daytime, independent from the type of lighting systems installed, is linked to later bed time, shorter time in bed and concomitant longer day-time alertness and positive emotions such as pleasure [62]. These findings lead to speculation of a dose-response relationship by which too little and too much power of light is detrimental whilst the beneficial dose is somewhere in between.

As for sleep problems, indoor bright-light interventions $(2,000-3,000$ lux ) as treatment for neuropsychiatric symptoms, including agitation, depression, dysphoria or apathy, showed similar ambiguous outcomes in dementia patients [55]. Indeed, bright light in the morning exacerbated agitation in patients with mild to moderate $\mathrm{AD}$ [63]. Agitation in those with severe AD was only minimal and therefore clinical irrelevant [64]. Forbes et al, (2014) concluded on the basis of the meta-analysis that there is no evidence that light intervention reduces neuropsychiatric symptoms or decreases the decline in cognition. The individual trials varied in the modalities of the light intervention but numbers were too small to allow for subgroup analyses that would determine, which modality (type, intensity, duration, time-of-day) might be beneficial for specific behaviours or types and severity of dementia.
A

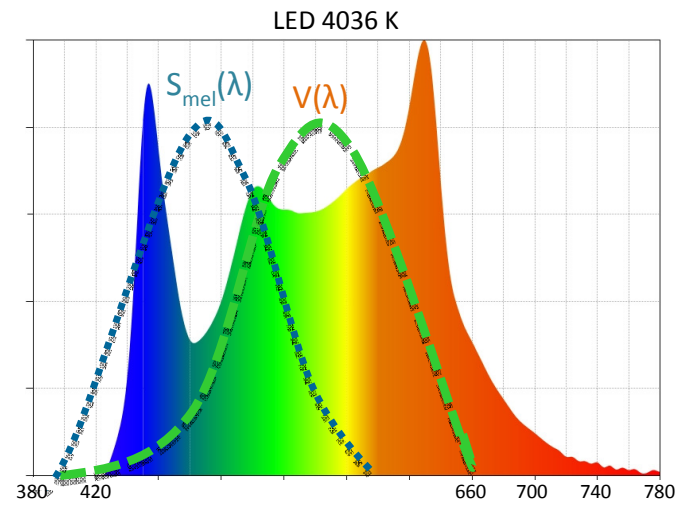

B

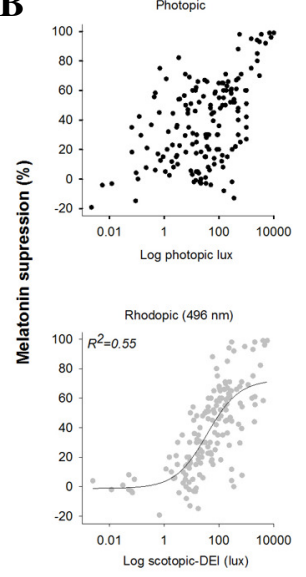

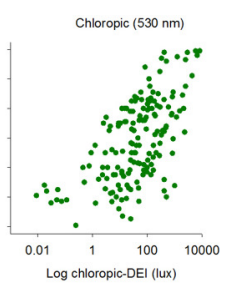

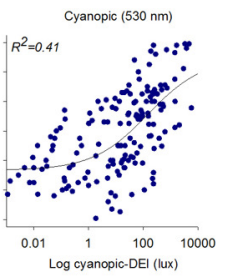

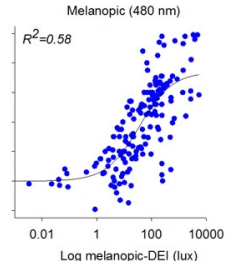

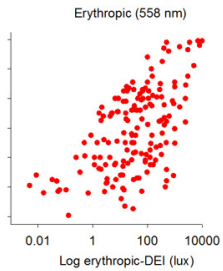

Figure 2. Properties of light as a potent stimulus for regulating visual image-forming and non-image forming functions. A) Schematic spectral power distribution of a LED with correlated colour temperature (CCT) of 4036K overlaid with the spectral sensitivity curves for melanopic pRGCs (blue-dotted, $S_{\text {mel }}[\lambda]$ ) and cone-driven photopic vision (green-dashed, V $[\lambda]$ ). Melanopic sensitivity covers wavelengths in the shorter ranges of light than photopic sensitivity, while a small proportion of the spectrum is shared by both. B) Biological effects of light, such as suppression of melatonin synthesis, are influenced by the spectral absorption curves of some photoreceptors in the eye. The dose-response curves illustrate the magnitude of melatonin suppression according to photoreceptor-weighted light intensities expressed as $\alpha$-opic daylight equivalent illuminance $(\alpha \mathrm{O}$ DEI) compared to photopic lux. The lines show the fit to a four parameter logistic model. Significant sigmoidal relationships between stimulus strength and melatonin suppression was found for cyanopic-, melanopic- and rhodopic-DEIs. The use of photopic lux showed no significant correlation. Photoreceptor-specific illuminances according to the colour of the spectrum to which they are most sensitive: Cyanopic: short-wave cones, Chloropic: medium-wave cones, Erythropic: long-wave cones, Melanopic: melanopsin, Rhodopic: rods. Scotopic: vision under dim light based on rods, Photopic: vision under well-lit light based on $\mathrm{s} / \mathrm{m}$ cones. Figure $2 \mathrm{~b}$ adapted from [68]. 
An architectural lighting system with programmable control of light intensity (2,500 lux) applied for an extended period resulted in staff complaining about visual discomfort [65]. Lower light levels (1250 lux) using the same system failed to show expected improvement in depression, agitated behaviour and sleep. Newer designs of lighting systems with programmable spectral content to deliver narrow band light targeting the peak response of the circadian system $(464 \mathrm{~nm}$ at $\sim 400$ lux vs $628 \mathrm{~nm}$ at $\sim 75 \mathrm{lux}$ ) allowed determining a clinically effective dose without excessive brightness [66]. In a small study this design induced significant reduction in cognitive decline at $464 \mathrm{~nm}$ compared to $628 \mathrm{~nm}$ light when administered in the morning.

Given the large impact of light on human well-being via melanopsin-based pathways on one hand and the accelerated growth of LED technology on the other, research on impact to guide technical standards is trailing behind of what is technically feasible. As a consequence, all published studies so far calculated efficacy of light in appropriately with a spectral luminous function $\mathrm{V}(\lambda)$ based on $555 \mathrm{~nm}$ for photopic vision (ISO 23539:2005(E)/CIE S 010/E:2004), and not with a function based on spectral distribution $\left(\mathrm{S}_{\mathrm{mel}}(\lambda)\right.$ in the range of $460-480 \mathrm{~nm}$ for the potent stimulation of the melanopsin-based system (Figure 2a). Hence, all previous results are skewed with respect to their effect size and need to be re-scaled. Researchers from chronobiology, physics and engineering aimed at a new light-measurement strategy taking into account the complex photoreceptive inputs to these non-image forming functions by redefining spectral luminous efficacy functions according to all five photoreceptors, so-called $\alpha$-opics [67, 68]. Large numbers of already published studies have been made comparable by rescaling light intensities to $\alpha$-opic daylight equivalent illuminances and indeed the biologically-weighted values showed better correlations with expected effects using, for example, melanopic-weighted rather than photopic light intensities for melatonin suppression (Figure 2b, [68]).

\section{CONCLUDING REMARKS}

Understanding the effects of light on human physiology and behaviour in patients with dementias requires systematic elucidation of complex neuronal systems in the eye and the brain, related to their functional roles in vision and basal physiology and behaviour. The pRGC population in the eye consists of several subtypes, each with different responses to light, suggesting possible specific functional roles for each subtype in NIF mediated behaviour, including sleep and mood. Progressive age-related impairments in the eye and brain may be in part responsible for the inefficient behavioural responses to artificial light interventions compared to the much stronger natural daylight. Ocular assessment (visual acuity, contrast sensitivity, colour vision, lens transmission, pupil size and reflex) and removal of cataract can improve the quality of live through better vision and sleep quality. Accurate information is important for vision (glare, acuity) and NIF functions (sleep latency and mood) and has to be taken into consideration for lighting solutions. Properties of current lighting systems are inflexible and not designed to take nonimage forming effects of light into account for older people's wellbeing. Previous bright light interventions in residential care homes did not live up to expected improvement in neuropsychiatric behaviours and sleep. However, fewer studies using daylight exposure have documented improvements for these behaviours in the elderly. Therefore, the future strategy for successful ambient light environments should consider to: 1) make use of daylight, 2) orientate on daylight quality (intensity and spectral composition) using spectral weighting functions and dynamic systems, 3) time of day-dependent modifications in light quality for maximum wellbeing. Recent innovative approaches in the lighting industry targeting pRGC requirements by adopting LED technology can potentially create health-oriented lighting systems with application for older individuals at home and in residential care homes. Metrics to calculate SIcompliant, photoreceptor-weighted light intensities have been developed and the alpha-opic daylight equivalent illuminances $(\alpha \mathrm{O}$-DEI) are expected to be more useful predictors for NIF effects than photopic quantities when using narrow spectral bands or broad spectrum light that includes strong peaks in the spectrum. Systematic measurements of image- (visual acuity, lens transmission, pupil size and reflex, visual comfort) and non-image forming responses (phase/amplitude of circadian marker rhythms, sleep, mood, cognition) to new lighting systems should use $\alpha \mathrm{O}$-DEIs to predict, assess, quantify, compare and reproduce potential impact of different light settings. Quantitative thresholds to promote or avoid certain NIF responses are urgently needed to provide guidance for lighting solutions in the nursing home sector.

\section{CONFLICT OF INTEREST}

The authors declare to have no conflict of interest in connection with this article.

\section{ACKNOWLEDGEMENTS}

The review was supported by Accelerate Solid State Lighting Innovation for Europe $\left(7^{\text {th }}\right.$ framework programme, 619249) and the National Institute for Health Research (NIHR) Oxford Biomedical Research Centre based at Oxford University Hospitals NHS Trust, Oxford University (A90305 and A92181). The views expressed are those of the author(s) and not necessarily those of the NHS, the NIHR, or the Department of Health. This study was in part supported by the infrastructure of the Sleep and Circadian 
Neuroscience Institute (SCNi). K. Wulff carried out the literature search and wrote the first draft of the manuscript. Both authors, K. Wulff and R.G. Foster, revised subsequent versions and approved the manuscript.

\section{REFERENCES}

[1] Balsalobre A, Damiola F, Schibler U. A serum shock induces circadian gene expression in mammalian tissue culture cells. Cell 93(6):929-37 (1998).

[2] Tosini G, Pozdeyev N, Sakamoto K, Iuvone PM. The circadian clock system in the mammalian retina. BioEssays : news and reviews in molecular, cellular and developmental biology 30(7):624-33 (2008).

[3] Freedman MS, Lucas RJ, Soni B, von Schantz M, Munoz M, David-Gray ZK, et al. Regulation of mammalian circadian behavior by non-rod, non-cone, ocular photoreceptors. Science 284:502-4 (1999).

[4] Gooley JJ, Lu J, Chou TC, Scammell TE, Saper CB. Melanopsin in cells of origin of the retinohypothalamic tract. Nat Neurosci 12:1165 (2001).

[5] Gooley JJ, Lu J, Fischer D, Saper CB. A broad role for melanopsin in nonvisual photoreception. $\mathbf{J}$ Neurosci 23(18):7093-106 (2003).

[6] Hannibal J, Hindersson P, Knudsen SM, Georg B, Fahrenkrug J. The photopigment melanopsin is exclusively present in pituitary adenylate cyclase-activating polypeptidecontaining retinal ganglion cells of the retinohypothalamic tract. J Neuroscience 22:RC191: 1-7 (2002).

[7] Hannibal J, Fahrenkrug J. Melanopsin: a novel photopigment involved in the photoentrainment of the brain's biological clock? 34(5):401-7 (2002).

[8] Panda S, Sato TK, Castrucci AM, Rollag MD, DeGrip WJ, Hogenesch JB, et al. Melanopsin (Opn4) requirement for normal light-induced circadian phase shifting. Science 298(5601):2213-6 (2002).

[9] Ruby NF, Brennan TJ, Xie X, Cao V, Franken P, Heller HC, et al. Role of melanopsin in circadian responses to light. Science 298(5601):2211-3 (2002).

[10] Berson DM, Dunn FA, Takao M. Phototransduction by retinal ganglion cells that set the circadian clock. Science 295:10703 (2002).

[11] Hattar S, Liao HW, Takao M, Berson DM, Yau KW. Melanopsin-containing retinal ganglion cells: architecture, projections, and intrinsic photosensitivity. Science 295:106570 (2002).

[12] Bowmaker JK, Dartnall HJ. Visual pigments of rods and cones in a human retina. J Physiol 298:501-11 (1980).

[13] Bondarenko LN. Spectral polarimetric measurements of the twighlight sky polarisation at the Zenith. Soviet Astronomy 8:299-302 (1964).

[14] Hader DP, Tevini,M. General Photobiology. Oxford: Pergamon Press; 1987. 323 p.

[15] Sekaran S, Foster RG, Lucas RJ, Hankins MW. Calcium imaging reveals a network of intrinsically light-sensitive inner-retinal neurons. Curr Biol 13(15):1290-8 (2003).

[16] Hattar S, Kumar M, Park A, Tong P, Tung J, Yau KW, et al. Central projections of melanopsin-expressing retinal ganglion cells in the mouse. The Journal of comparative neurology 497(3):326-49 (2006).

[17] Schmidt TM, Do MT, Dacey D, Lucas R, Hattar S, Matynia A. Melanopsin-positive intrinsically photosensitive retinal ganglion cells: from form to function. $\mathrm{J}$ Neurosci 31(45):16094-101 (2011).
[18] Schmidt TM, Chen SK, Hattar S. Intrinsically photosensitive retinal ganglion cells: many subtypes, diverse functions. Trends in neurosciences 34(11):572-80 (2011).

[19] Hughes S, Rodgers J, Hickey D, Foster RG, Peirson SN, Hankins MW. Characterisation of light responses in the retina of mice lacking principle components of rod, cone and melanopsin phototransduction signalling pathways. Scientific reports 6:28086 (2016).

[20] Nayak SK, Jegla T, Panda S. Role of a novel photopigment, melanopsin, in behavioral adaptation to light. Cellular and molecular life sciences : CMLS 64(2):144-54 (2007).

[21] Pilorz V, Tam SK, Hughes S, Pothecary CA, Jagannath A, Hankins MW, et al. Melanopsin Regulates Both SleepPromoting and Arousal-Promoting Responses to Light. PLoS biology 14(6):e1002482 (2016).

[22] Zaidi FH, Hull JT, Peirson SN, Wulff K, Aeschbach D, Gooley JJ, et al. Short-wavelength light sensitivity of circadian, pupillary, and visual awareness in humans lacking an outer retina. Curr Biol 17(24):2122-8 (2007).

[23] Storchi R, Milosavljevic N, Eleftheriou CG, Martial FP Orlowska-Feuer P, Bedford RA, et al. Melanopsin-driven increases in maintained activity enhance thalamic visual response reliability across a simulated dawn. Proc Natl Acad Sci U S A 112(42):E5734-43 (2015).

[24] Cuthbertson FM, Peirson SN, Wulff K, Foster RG, Downes SM. Blue light-filtering intraocular lenses: review of potential benefits and side effects. J Cataract Refract Surg 35(7):128197 (2009).

[25] van de Kraats J, van Norren D. Optical density of the aging human ocular media in the visible and the UV. Journal of the Optical Society of America A, Optics, image science, and vision 24(7): 1842-57 (2007)

[26] Winn B, Whitaker D, Elliott DB, Phillips NJ. Factors affecting light-adapted pupil size in normal human subjects. Investigative ophthalmology \& visual science 35(3):1132-7 (1994).

[27] Veitch JA. Psychological processes influencing lighting quality. Journal of the Illuminating Engineering Society 30(1) (2001).

[28] Bron TAV, G.F.;Koretz,J.;Maraini,J.;Harding,J.J. The aging lens. Ophthalmologica 214:86-104 (2000).

[29] Najjar RP, Chiquet C, Teikari P, Cornut PL, Claustrat B, Denis $\mathrm{P}$, et al. Aging of non-visual spectral sensitivity to light in humans: compensatory mechanisms? PLoS One 9(1):e85837 (2014).

[30] Alexander I, Cuthbertson FM, Ratnarajan G, Safa R, Mellington FE, Foster RG, et al. Impact of cataract surgery on sleep in patients receiving either ultraviolet-blocking or bluefiltering intraocular lens implants. Investigative ophthalmology \& visual science 55(8):4999-5004 (2014).

[31] Lerner AD, S.; Belkin,J.; Lass, J.; Riedel,T.; Steinemann,T.; Sami,S.; Gilmore,G. Viusal and cognitive improvement following cataract surgery in subjects with dementia. Alzheimer's Association International Conference; Copenhagen: The Journal of Alzheimer's Association; 2014. p. P456-7.

[32] Armstrong RA. Alzheimer's disease and the eye. Journal of Optometry 2:103-11 (2009).

[33] Parisi V, Restuccia R, Fattapposta F, Mina C, Bucci MG, Pierelli F. Morphological and functional retinal impairment in Alzheimer's disease patients. Clinical neurophysiology : official journal of the International Federation of Clinical Neurophysiology 112(10):1860-7 (2001).

[34] Hinton DR, Sadun AA, Blanks JC, Miller CA. Optic-nerve degeneration in Alzheimer's disease. The New England journal of medicine 315(8):485-7 (1986). 
[35] Sadun AA, Bassi CJ. Optic nerve damage in Alzheimer's disease. Ophthalmology 97(1):9-17 (1990).

[36] Tsai CS, Ritch R, Schwartz B, Lee SS, Miller NR, Chi T, et al. Optic nerve head and nerve fiber layer in Alzheimer's disease. Archives of ophthalmology 109(2):199-204 (1991).

[37] Goldstein LE, Muffat JA, Cherny RA, Moir RD, Ericsson $\mathrm{MH}$, Huang $\mathrm{X}$, et al. Cytosolic beta-amyloid deposition and supranuclear cataracts in lenses from people with Alzheimer's disease. Lancet 361(9365): 1258-65 (2003).

[38] Scheuermaier K, Laffan AM, Duffy JF. Light exposure patterns in healthy older and young adults. J Biol Rhythms 25(2):113-22 (2010).

[39] Wright KP, Jr., McHill AW, Birks BR, Griffin BR, Rusterholz T, Chinoy ED. Entrainment of the human circadian clock to the natural light-dark cycle. Curr Biol 23(16):1554-8 (2013).

[40] Higuchi S, Lee SI, Kozaki T, Harada T, Tanaka I. Late circadian phase in adults and children is correlated with use of high color temperature light at home at night. Chronobiol Int 33(4):448-52 (2016).

[41] Obayashi K, Saeki K, Iwamoto J, Okamoto N, Tomioka K, Nezu S, et al. Positive effect of daylight exposure on nocturnal urinary melatonin excretion in the elderly: a crosssectional analysis of the HEIJO-KYO study. J Clin Endocrinol Metab 97(11):4166-73 (2012).

[42] Chang AM, Santhi N, St Hilaire M, Gronfier C, Bradstreet DS, Duffy JF, et al. Human responses to bright light of different durations. J Physiol 590(Pt 13):3103-12 (2012).

[43] St Hilaire MA, Gooley JJ, Khalsa SB, Kronauer RE, Czeisler CA, Lockley SW. Human phase response curve to a $1 \mathrm{~h}$ pulse of bright white light. J Physiol 590(Pt 13):3035-45 (2012).

[44] Kim SJ, Benloucif S, Reid KJ, Weintraub S, Kennedy N, Wolfe LF, et al. Phase-shifting response to light in older adults. J Physiol 592(Pt 1):189-202 (2014).

[45] Duffy JF, Zeitzer JM, Czeisler CA. Decreased sensitivity to phase-delaying effects of moderate intensity light in older subjects. Neurobiology of aging 28(5):799-807 (2007).

[46] Herljevic M, Middleton B, Thapan K, Skene DJ. Lightinduced melatonin suppression: age-related reduction in response to short wavelength light. Experimental gerontology 40(3):237-42 (2005).

[47] Wehr TA, Giesen HA, Schulz PM, Anderson JL, JosephVanderpool JR, Kelly K, et al. Contrasts between symptoms of summer depression and winter depression. J Affect Disord 23(4):173-83 (1991).

[48] Lee TM, Chan CC. Dose-response relationship of phototherapy for seasonal affective disorder: a meta-analysis. Acta psychiatrica Scandinavica 99(5):315-23 (1999).

[49] Tuunainen A, Kripke DF, Endo T. Light therapy for nonseasonal depression. The Cochrane database of systematic reviews (2):CD004050 (2004)

[50] Martiny K, Lunde M, Unden M, Dam H, Bech P. Adjunctive bright light in non-seasonal major depression: results from clinician-rated depression scales. Acta psychiatrica Scandinavica 112(2):117-25 (2005).

[51] Sondergaard MP, Jarden JO, Martiny K, Andersen G, Bech P. Dose response to adjunctive light therapy in citalopramtreated patients with post-stroke depression. A randomised, double-blind pilot study. Psychotherapy and psychosomatics 75(4):244-8 (2006).

[52] Golden RN, Gaynes BN, Ekstrom RD, Hamer RM, Jacobsen FM, Suppes T, et al. The efficacy of light therapy in the treatment of mood disorders: a review and meta-analysis of the evidence. Am J Psychiatry 162(4):656-62 (2005).
[53] Genhart MJ, Kelly KA, Coursey RD, Datiles M, Rosenthal NE. Effects of bright light on mood in normal elderly women. Psychiatry Res 47(1):87-97 (1993).

[54] Montgomery P, Dennis J. Bright light therapy for sleep problems in adults aged $60+$. The Cochrane database of systematic reviews (2):CD003403 (2002).

[55] Forbes D, Blake CM, Thiessen EJ, Peacock S, Hawranik P. Light therapy for improving cognition, activities of daily living, sleep, challenging behaviour, and psychiatric disturbances in dementia. The Cochrane database of systematic reviews 2:CD003946 (2014).

[56] Van Someren EJ, Kessler A, Mirmiran M, Swaab DF. Indirect bright light improves circadian rest-activity rhythm disturbances in demented patients. Biol Psychiatry 41(9):95563 (1997).

[57] Van Someren EJ, Swaab DF, Colenda CC, Cohen W, McCall WV, Rosenquist PB. Bright light therapy: improved sensitivity to its effects on rest-activity rhythms in Alzheimer patients by application of nonparametric methods. Chronobiol Int 16(4):505-18 (1999).

[58] Riemersma-van der Lek RF, Swaab DF, Twisk J, Hol EM, Hoogendijk WJ, Van Someren EJ. Effect of bright light and melatonin on cognitive and noncognitive function in elderly residents of group care facilities: a randomized controlled trial. Jama 299(22):2642-55 (2008).

[59] Salami O, Lyketsos C, Rao V. Treatment of sleep disturbance in Alzheimer's dementia. International journal of geriatric psychiatry 26(8):771-82 (2011).

[60] van Maanen A, Meijer AM, van der Heijden KB, Oort FJ. The effects of light therapy on sleep problems: A systematic review and meta-analysis. Sleep Med Rev 29:52-62 (2016).

[61] Hopkins SM, P.L.; Schlangen, L.J.M.; Williams, P.; Skene, D.J.; Middleton, B. Blue-enriched Lighting for Older People Living in Care Homes: Effect on Activity, Actigraphic Sleep, Mood and Alertness. Current Alzheimer's Research in press.

[62] Münch MS, M; Bieler, K; Goldbach, R; Fuhrmann, T; Zumstein, N; Vonmoos, P; Scartezzini, JL; Wirz-Justice, A; Cajochen C. Bright light delights: effects of daily light exposure on emotions, rest-activity cycles, sleep and melatonin secretion in severely demented patients. Current Alzheimer's Research in press.

[63] Barrick AL, Sloane PD, Williams CS, Mitchell CM, Connell BR, Wood W, et al. Impact of ambient bright light on agitation in dementia. International journal of geriatric psychiatry 25(10):1013-21 (2010).

[64] Dowling GA, Graf CL, Hubbard EM, Luxenberg JS. Light treatment for neuropsychiatric behaviors in Alzheimer's disease. Western journal of nursing research 29(8):961-75 (2007).

[65] White MD, Ancoli-Israel S, Wilson RR. Senior living environments: evidence-based lighting design strategies. Herd 7(1):60-78 (2013)

[66] Royer M, Ballentine NH, Eslinger PJ, Houser K, Mistrick R, Behr R, et al. Light therapy for seniors in long term care. Journal of the American Medical Directors Association 13(2):100-2 (2012).

[67] Lucas RJ, Peirson SN, Berson DM, Brown TM, Cooper HM, Czeisler CA, et al. Measuring and using light in the melanopsin age. Trends in neurosciences 37(1):1-9 (2014).

[68] Gimenez MS; Schlangen L, Lang D, Beersma LED, Novotny P, Plischke H, Wulff K, Linek M, Cajochen C, Loeffler J, Lasauskaite R, Bhusal P, Halonen L. D3.7 Report on metric to quantify biological light exposure doses. Accelerate SSL Innovation for Europe. Brussels: European Commission, Deliverable 3.7 FP7-ICT-2013-11-619240 (2016). 
